\title{
Spin-orbit coupling and crystal-field splitting in the electronic and optical properties of nitride quantum dots with a wurtzite crystal structure
}

\author{
S. Schulz and G. Czycholl \\ Institute for Theoretical Physics, University of Bremen, 28359 Bremen, Germany \\ S. Schumacher \\ College of Optical Sciences, University of Arizona, Tucson, Arizona 85721, USA
}

(Dated: October 25, 2018)

\begin{abstract}
We present an $s p^{3}$ tight-binding model for the calculation of the electronic and optical properties of wurtzite semiconductor quantum dots (QDs). The tight-binding model takes into account strain, piezoelectricity, spin-orbit coupling and crystal-field splitting. Excitonic absorption spectra are calculated using the configuration interaction scheme. We study the electronic and optical properties of InN/GaN QDs and their dependence on structural properties, crystal-field splitting, and spinorbit coupling.

PACS numbers: 78.67.Hc, 73.22.Dj, 71.35.-y
\end{abstract}

\section{INTRODUCTION}

Group-III nitrides have shown great potential in optoelectronic devices with a wide range of applications $\frac{1}{-}$ InGaN/GaN quantum wells typically constitute the active region in light-emitting diodes and laser diodes, covering a wide spectral range from near ultraviolet to infrared. In particular, devices with an active region based on pure or almost pure $\mathrm{InN}$ are of great interest to reach operational frequencies in the infrared spectral range. 2 The small InN band gap $(0.7-0.8 \mathrm{eV})^{\frac{3}{3}}$ can be extremely useful for telecommunication-wavelength devices. The combination of this inherent property of $\mathrm{InN}$ with the self-assembly of nanostructures based on this material, provides further possibilities for useful future optoelectronic devices. In particular, quantum dot (QD) structures are promising candidates, acting as electron-hole recombination centers increasing the emission efficiency. These zero-dimensional nanostructures also have the potential to act as single-photon emitting devices $\underline{\underline{4}}$

This work is dedicated to the investigation of the electronic and optical properties of self-assembled InN QDs. The present study is based on a fully atomistic empirical tight-binding model. In contrast to multi-band $\mathbf{k} \cdot \mathbf{p}$ approaches it takes into account the structure of the underlying atomic lattice and is capable to describe the electronic wave functions beyond an envelope function approximation. Our model includes strain effects and electrostatic built-in fields, which are of major importance in group-III nitride based nanostructures with an underlying wurtzite crystal structure. In this paper, special attention is paid to the possible influence of the weak crystal-field (CF) splitting and weak spinorbit ( $\mathrm{SO}$ ) coupling on the localized single-particle wave functions as well as on the optical properties of the investigated structures. These effects have commonly been neglected in previous studies, some of which were dedicated to GaN/AlN nanostructures 5.6.7.8, others to $\mathrm{InN} / \mathrm{GaN}$ nanostrucutres $9,10,11,12,13,14$. Since in these materials both effects are merely of the order of a few meV (Ref $\left.\frac{3}{\underline{3}}\right)$, this approximation can be well justified.

On a more fundamental level the resulting symmetry properties dictated by spatial and spin degrees of freedom and, taking SO coupling into account, by the coupled influence of both, have the potential to support or lift certain degeneracies in the electronic energy spectra. Based on group-theoretical arguments we discuss that in the system under investigation for both electrons and holes, at most two-fold degeneracies are supported. This is especially of interest with regard to a recent discussion of the energy level structure in semiconductor QDs with a zinc blende structure ${ }^{15}$ and recent results for wurtzite In$\mathrm{GaN} / \mathrm{GaN}$ QDs obtained within an 8-band $\mathbf{k} \cdot \mathbf{p}$ model ${ }^{16}$. Following these general group-theoretical arguments, our numerical results show that SO coupling and CF splitting slightly change the results for the InN/GaN QD system under investigation. However, the additional splittings in the electronic energy shell structure caused by the SO coupling are at most of the order of a few meV. From this, no significant qualitative changes of the optical properties are found: The excitonic absorption lines show only very small additional splittings and the interband dipole selection rules are basically unaltered.

\section{THEORY AND QUANTUM DOT MODEL}

\section{A. The Tight-Binding Model}

For the investigation of the single particle states in small QD structures, the description by a multi-band approach is required. In order to take into account the underlying wurtzite structure of the structures under investigation, we choose a microscopic $s p^{3}$ tight-binding (TB) model. The general aspects of the TB model are discussed in detail in Ref ${ }^{12}$. Here, we briefly summarize the main ingredients of this model, and focus our attention on the role of crystal-field splitting and spin-orbit 


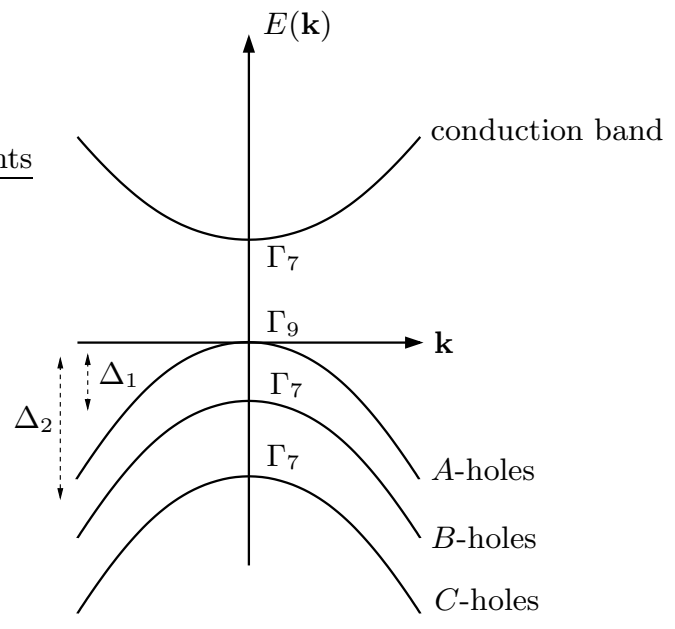

FIG. 1: Schematic band structure of wurtzite semiconductors with conduction band and three valence bands. The valence band splittings introduced by crystal field splitting and spinorbit coupling are denoted by $\Delta_{1}$ and $\Delta_{2}$, respectively. $\Delta_{1}$ and $\Delta_{2}$ are calculated according to Eq. (1). Additionally, the symmetries of the irreducible representations $\Gamma_{i}$ are given.

coupling.

In the $s p^{3}$ TB model of Ref $\frac{12}{2}$ the relevant electronic structure of anions and cations is, for each spin orientation, described by the outermost valence orbitals, $s$, $p_{x}, p_{y}$ and $p_{z}$, and the overlap of these basis orbitals is restricted to nearest neighbors. Being only of the order of a few meV, so far, the influence of CF splitting and SO coupling has been neglected in the model. In the present work, we extend our TB mode 12 to introduce these two contributions and investigate their possible influence on the electronic single-particle states and energies in $\mathrm{InN} / \mathrm{GaN}$ QD systems.

The combination of $\mathrm{CF}$ splitting and SO interaction leads to a so-called three-edge structure in the vicinity of the $\Gamma$ point. The top of the resulting valence band structure is commonly labeled as $A, B$, and $C$ bands in order of increasing energy. This three-edge structure is schematically shown in Fig. 1. Two of these three bands are of $\Gamma_{7}$ and one of $\Gamma_{9}$ symmetry. To describe this band structure in the vicinity of the $\Gamma$ point in the framework of a TB model one has to take into account both $\mathrm{CF}$ splitting and SO interaction.

The SO coupling is included as outlined by Chadi in $\operatorname{Re} \mathrm{fl}^{18}$. Due to the high ionicity of the bonds in the nitride system $\frac{19}{}$, the contribution of the SO coupling to the valence band structure is dominated by the anion contributions. Therefore, by introducing the parameter $\lambda$, we include spin-orbit coupling at the anion sites only. The parameter $\lambda$ is used to reproduce the correct splitting $\Delta_{1}$ of the valence bands $A$ and $B$.

As discussed in Ref $\stackrel{17}{17}$, the small CF splitting $\Delta_{\text {cf }}$ of the wurtzite crystal differentiates the $p_{z}$ orbital from the $p_{x}$ and $p_{y}$ orbitals. Pseudo-potential calculations in local density approximation indicate that for the studied
TABLE I: Tight-binding parameters (in eV) for the nearest neighbors of wurtzite InN and GaN. The notation of Ref ${ }^{17}$ is used.

\begin{tabular}{|l|c|c|c||c|c|c|}
\hline \hline & \multicolumn{3}{|c||}{$\mathrm{InN}[\mathrm{eV}]$} & \multicolumn{3}{c|}{$\mathrm{GaN}[\mathrm{eV}]$} \\
\hline & $\Delta_{\text {cf }}=0$ & $\Delta_{\mathrm{cf}} \neq 0$ & $\Delta_{\mathrm{cf}} \neq 0$ & $\Delta_{\text {cf }}=0$ & $\Delta_{\text {cf }} \neq 0$ & $\Delta_{\text {cf }} \neq 0$ \\
& $\Delta_{\text {so }}=0$ & $\Delta_{\text {so }}=0$ & $\Delta_{\text {so }} \neq 0$ & $\Delta_{\text {so }}=0$ & $\Delta_{\text {so }}=0$ & $\Delta_{\text {so }} \neq 0$ \\
\hline $\mathrm{E}(\mathrm{s}, \mathrm{a})$ & -6.791 & -6.5134 & -6.6046 & -11.012 & -8.9893 & -8.5282 \\
$\mathrm{E}(\mathrm{p}, \mathrm{a})$ & 0.000 & 0.0000 & 0.0000 & 0.005 & 0.0015 & -0.0024 \\
$\mathrm{E}\left(\mathrm{p}_{z}, \mathrm{a}\right)$ & 0.000 & -0.0418 & -0.0400 & 0.005 & -0.0203 & -0.0208 \\
$\mathrm{E}(\mathrm{s}, \mathrm{c})$ & -3.015 & -3.3923 & -3.3500 & 1.438 & 0.7851 & 0.6945 \\
$\mathrm{E}(\mathrm{p}, \mathrm{c})$ & 8.822 & 8.8220 & 8.8203 & 10.896 & 10.0986 & 10.0996 \\
$\mathrm{~V}(\mathrm{~s}, \mathrm{~s})$ & -5.371 & -5.5267 & -5.5330 & -5.318 & -5.6918 & -5.6808 \\
$\mathrm{~V}(\mathrm{x}, \mathrm{x})$ & 0.022 & 0.0156 & 0.1221 & -0.222 & -0.1223 & -0.0699 \\
$\mathrm{~V}(\mathrm{x}, \mathrm{y})$ & 6.373 & 6.3794 & 6.2772 & 7.136 & 6.7902 & 6.7328 \\
$\mathrm{~V}(\mathrm{sa}, \mathrm{pc})$ & 0.370 & 0.9576 & 0.9307 & 0.628 & 0.2641 & 1.3633 \\
$\mathrm{~V}(\mathrm{pa}, \mathrm{sc})$ & 7.5 & 7.5574 & 7.4136 & 7.279 & 8.0324 & 7.7173 \\
$\lambda$ & 0 & 0 & 0.0016 & 0 & 0 & 0.0023 \\
\hline \hline
\end{tabular}

materials the bulk crystal field splitting between the $A$ and $C$ valence bands, schematically shown in Fig. 2 , cannot be reproduced from first principles, unless thirdnearest-neighbor interactions are taken into account ${ }^{20}$. The TB model discussed in Ref $\frac{12}{\underline{12}}$ considers only nearestneighbor hopping matrix elements and treats the four nearest neighbor atoms as equivalent. To account for the $A-C$ splitting within the empirical $s p^{3}$ TB model with nearest-neighbor coupling, we introduce the additional parameter $E_{p_{z}, p_{z}}^{A}$ on the anion sites for the on-site matrix elements of the $p_{z}$ orbitals. This additional term is used to reproduce the splitting $\Delta_{2}$ of the $A-C$ bands at the zone center $\Gamma$.

With four atoms per unit cell, the resulting Hamiltonian is a $32 \times 32$ matrix for each $\mathbf{k}$-point. This Hamiltonian parametrically depends on the different TB matrix elements. The parameters given in Ref $\underline{12}$ have been

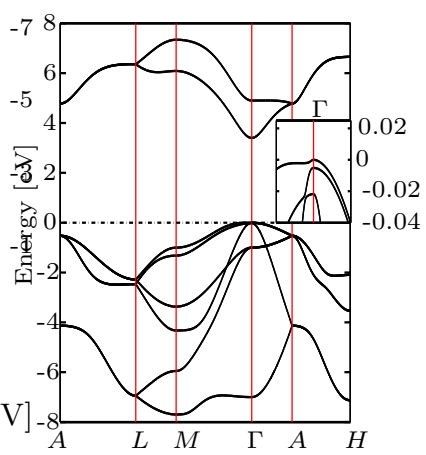

(a) GaN band structure

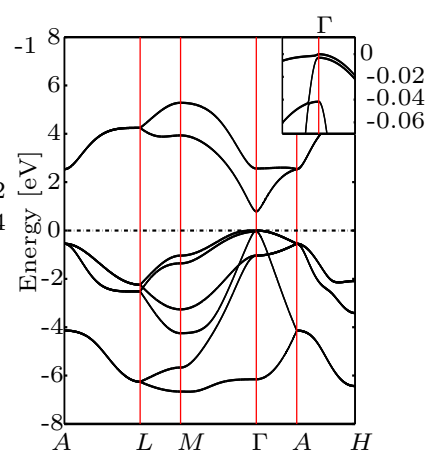

(b) InN band structure
FIG. 2: (Color online) Bulk band structure of wurtzite (a) InN and (b) GaN obtained using an $s p^{3} \mathrm{~TB}$ model including crystal field splitting and spin-orbit coupling. The insets show the three-edge valence band structure in the vicinity of the $\Gamma$ point. 
re-calculated to reproduce the three-edge structure the wurtzite band structure $\frac{3,21,22}{1}$ in the vicinity of the $\Gamma$ point. The additional matrix elements $\lambda$ and $E_{p_{z}, p_{z}}^{A}$ are adjusted to reproduce the splittings between the different valence bands ( $A, B$ and $C$ bands), which are given by ${ }^{23}$

$$
\Delta_{1,2}=\left(\frac{\Delta_{\mathrm{so}}+\Delta_{\mathrm{cf}}}{2}\right) \mp \sqrt{\left(\frac{\Delta_{\mathrm{so}}+\Delta_{\mathrm{cf}}}{2}\right)^{2}-\frac{2}{3} \Delta_{\mathrm{so}} \Delta_{\mathrm{cf}}},
$$

where SO and CF splitting are denoted as $\Delta_{\text {so }}$ and $\Delta_{\text {cf }}$ respectively. Table \ summarizes the resulting TB parameters. The bulk band structures obtained from these parameters are shown in Fig. 2 for GaN and $\mathrm{InN}$. The 'complicated' valence band structure in the vicinity of the $\Gamma$ point shows very good agreement with other TB models 24 (cf. insets of Fig. 2), ab-initio approaches 25 , pseudo potential and $\mathbf{k} \cdot \mathbf{p}$ calculations ${ }^{26}$.

\section{B. Geometry of the Quantum Dot Structure}

In the framework of a TB model, the QD is modeled on an atomistic level. The TB parameters at each atom site $\mathbf{R}$ of the underlying wurtzite lattice are set according to the bulk values of the respective occupying atom. In the following, we consider lens-shaped InN QDs, grown in $c$ direction and residing on an InN wetting layer (WL). The entire structure is embedded inside a GaN matrix. For the numerical calculations, a finite cell (box) with a wurtzite lattice and fixed (zero) boundary conditions is used. The cell is sufficiently large to avoid numerical artifacts in the bound single particle states due to the cell boundaries (in particular, no artifacts from the artificial cubic symmetry of the supercell are found). In the following we discuss three different QD sizes with diameters $d=4.5,5.7,7.7 \mathrm{~nm}$ and heights $h=1.6,2.3,3.0 \mathrm{~nm}$, respectively. The WL thickness is one lattice constant $c$.

In a first step we neglect strain induced displacements of the atoms and concentrate on effects which can exclusively be attributed to crystal-field splitting and spinorbit coupling. The influence of strain effects will be discussed separately in Sec. IIB

In contrast to semiconductor heterostructures with a zinc blende structure, the III-V wurtzite nitrides exhibit a considerably larger built-in electrostatic field 27 . In order to account for this field in our model, the electrostatic potential $\phi_{p}(\mathbf{r})$ is obtained from the solution of the Poisson equation and enters as a site-diagonal contribution $V_{p}(\mathbf{r})=-e \phi(\mathbf{r})$ to the TB Hamiltonian 10 . The polarization $\mathbf{P}$ has two contributions, the spontaneous polarization $\mathbf{P}^{\text {spont }}$ and the piezo-electric contribution $\mathbf{P}^{\text {piezo }}$ caused by strain inside the system. For the latter we apply the approximation described in Ref. $\frac{28}{}$ and assume $\mathbf{P}^{\text {piezo }} \sim \mathbf{e}_{z}$, which is a reasonable approximation for the considered QD geometry $\underline{\underline{10}}$. Further details of this procedure are given in Ref $\frac{12}{2}$.

\section{Many-Body Hamiltonian, Coulomb and Dipole Matrix Elements}

Having discussed the TB Hamiltonian used for the calculation of the bound single-particle states, we now turn our attention to the investigation of the optical properties of the studied QD system. We start with the following Hamiltonian $H$ that describes the dynamics of the interacting charge carriers in the system:

$$
H=H_{0}+H_{C}+H_{D}
$$

This Hamiltonian consists of three parts and is given in the basis of the QD one-particle eigenstates. The contribution $H_{0}$

$$
H_{0}=\sum_{i} \epsilon_{i}^{e} c_{i}^{\dagger} c_{i}+\sum_{i} \epsilon_{i}^{h} h_{i}^{\dagger} h_{i}
$$

is the one-particle part, which is diagonal in the chosen basis,

$$
\begin{aligned}
H_{C}= & \frac{1}{2} \sum_{i j k l} V_{i j, k l}^{e e} c_{i}^{\dagger} c_{j}^{\dagger} c_{k} c_{l}+\frac{1}{2} \sum_{i j k l} V_{i j, k l}^{h h} h_{i}^{\dagger} h_{j}^{\dagger} h_{k} h_{l} \\
& -\sum_{i j k l} V_{i j, k l}^{h e} h_{i}^{\dagger} c_{j}^{\dagger} c_{k} h_{l},
\end{aligned}
$$

describes the Coulomb interaction of electrons (e) in the conduction band states and holes (h) in the valence band states, and

$$
H_{D}=\sum_{i, j}\left(e\langle i|\mathbf{E r}| j\rangle c_{i} h_{j}+\text { h.c. }\right),
$$

includes the coupling of the electronic system to an external electromagnetic field $\mathbf{E}$ in dipole approximation. The creation and annihilation operators for electrons (holes) in the single-particle state $|i\rangle$ with energy $\epsilon_{i}^{e}\left(\epsilon_{i}^{h}\right)$ are denoted by $c_{i}^{\dagger}\left(h_{i}^{\dagger}\right)$ and $c_{i}\left(h_{i}\right)$, respectively. The Coulomb interaction matrix elements are labeled by $V_{i j k l}^{\lambda \lambda^{\prime}}$.

The calculation of the Coulomb interaction matrix elements requires - at least in principle - the knowledge of the localized basis states implicitly underlying the TB wave functions. However, since the Coulomb matrix elements are dominated by the long-range character of the interaction, in the calculation of these matrix elements the charge densities in the localized orbitals are approximated by point charges. A more detailed discussion of this issue is given in Ref ${ }^{12}$. This approximation leads to the following explicit form of the Coulomb matrix elements:

$$
V_{i j k l}=\sum_{\mathbf{R R}^{\prime}} \sum_{\alpha \beta} c_{\mathbf{R}_{\alpha}}^{i *} c_{\mathbf{R}^{\prime} \beta}^{j *} c_{\mathbf{R}^{\prime} \beta}^{k} c_{\mathbf{R} \alpha}^{l} V\left(\mathbf{R}-\mathbf{R}^{\prime}\right),
$$

with

$$
V\left(\mathbf{R}-\mathbf{R}^{\prime}\right)=\frac{e_{0}^{2}}{4 \pi \varepsilon_{0} \varepsilon_{r}\left|\mathbf{R}-\mathbf{R}^{\prime}\right|} \quad \text { for } \quad \mathbf{R} \neq \mathbf{R}^{\prime}
$$

and

$$
V(0)=\frac{1}{V_{u c}^{2}} \int_{u c} d^{3} r d^{3} r^{\prime} \frac{e_{0}^{2}}{4 \pi \varepsilon_{0} \varepsilon_{r}\left|\mathbf{r}-\mathbf{r}^{\prime}\right|} \approx V_{0} .
$$


The expansion coefficients $c_{\alpha, \mathbf{R}}^{i}$ are related to the $\mathrm{i}^{\text {th }}$ oneparticle wave function $\Phi_{i}(\mathbf{r})=\sum_{\alpha, \mathbf{R}} c_{\alpha, \mathbf{R}}^{i} \phi_{\alpha, \mathbf{R}}(\mathbf{r})$ where $\phi_{\alpha, \mathbf{R}}(\mathbf{r})$ denotes the atomic wave functions localized at the lattice site $\mathbf{R}$.

To calculate the dipole matrix elements $\mathbf{d}_{i j}^{e h} \propto$ $\left\langle\psi_{i}^{e}|\mathbf{r}| \psi_{j}^{h}\right\rangle$ entering Eq. (44), we use numerically orthogonalized Slater orbitals ${ }^{12}$ to account for the short-range character of the dipole operator. The numerically orthogonalized Slater orbitals fulfill the basic properties of the localized basis states underlying the TB model: symmetry, spatial orientation 29 , and orthogonality. We also include the anion-cation structure of the crystal and the slight nonlocality of the dipole operator by including contributions from up to second nearest neighbors.

In analogy to the bulk systems, a separation of the orbital and spin part (both included in the index $\alpha$ ) is prohibited by the spin-orbit coupling. In contrast to the bulk case, as will be discussed in the following section, the strong band mixing prevents a strict classification of QD single-particle states according to their angular momentum. Therefore, even total angular momentum selection rules are no longer applicable. Ignoring the band mixing characteristics in the zero-dimensional structures, any treatment of many-body effects based on strict selection rules for the total angular momentum, yields inaccurate predictions of level degeneracies. However, as discussed in detail in Ref. ${ }^{13}$, the selection rules can always be analyzed on symmetry grounds.

With the dipole and Coulomb matrix elements and the many-body Hamiltonian, given in Eq. (2), the calculation of optical spectra can be carried out as described in Refs $\stackrel{13,30}{ }$.

\section{RESULTS FOR LENS-SHAPED InN QUANTUM DOTS}

\section{A. Single Particle states and Energies}

Having determined the TB parameters, the singleparticle states and energies of the three different QDs discussed in Sec. IIB can be calculated.

First we turn our attention to the single particle states of the large QD $(d=7.7 \mathrm{~nm}, h=3.0 \mathrm{~nm})$. In order to assess the impact of the CF splitting and SO coupling, we have performed our calculations in three steps. In a first step we neglect both SO interaction and CF effects $\left(\Delta_{\mathrm{so}}=0, \Delta_{\mathrm{cf}}=0\right)$. In this case we are left with the $s p^{3}$ TB model discussed in Ref. ${ }^{12}$. In step two we introduce only the crystal field splitting $\left(\Delta_{\mathrm{so}}=0, \Delta_{\mathrm{cf}} \neq 0\right)$, by including the additional parameter $E_{p_{z}, p_{z}}^{A}$ in the TB model. In the final step both contributions are taken into account $\left(\Delta_{\text {so }} \neq 0, \Delta_{\text {cf }} \neq 0\right)$. In each step the TB parameters are re-optimized in such a way that band gap and the energetic positions of other bands of the wurtzite bulk band structure at the $\Gamma$ point are reproduced. In other words, in the first step $\left(\Delta_{\text {so }}=\Delta_{\text {cf }}=0\right)$ we use the parameters

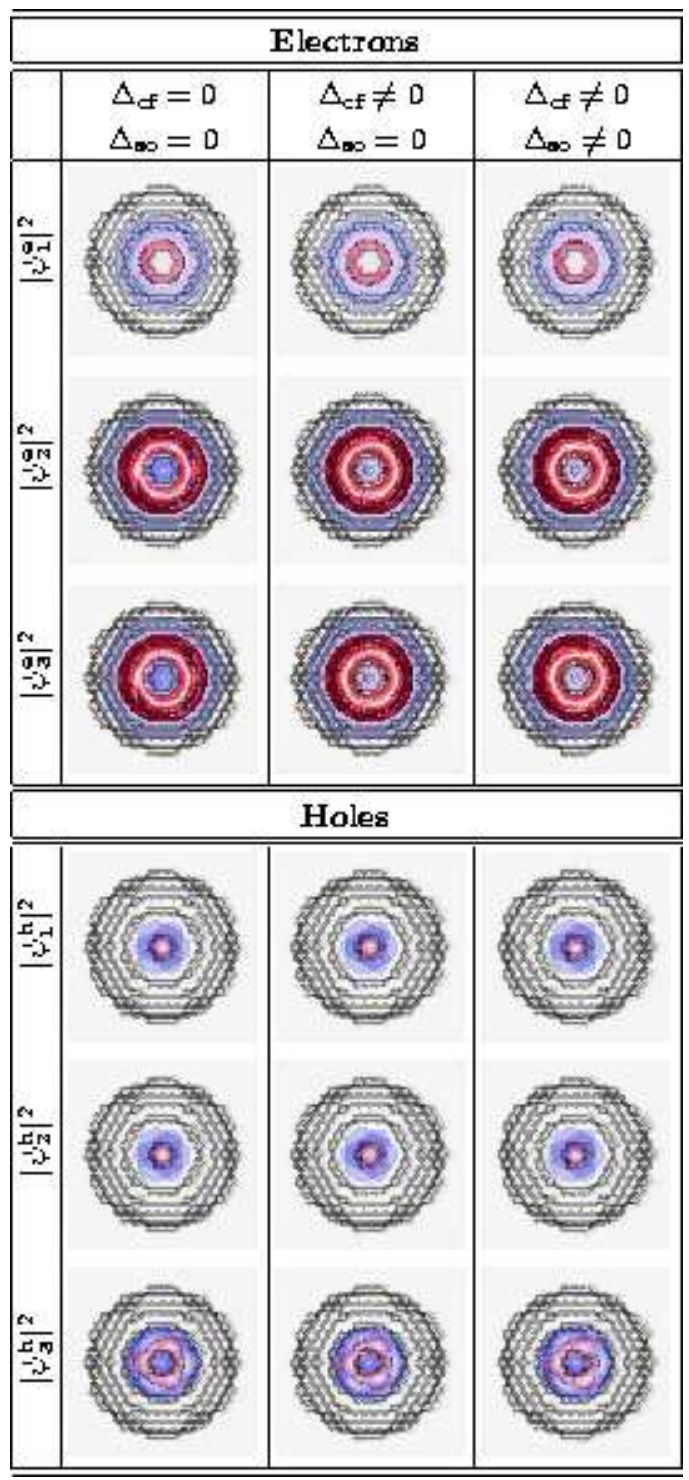

FIG. 3: (Color online) Top view of the large lens-shaped InN QD structure with the first three bound states for electrons (upper part) and holes (lower part). Depicted are isosurfaces of the probability density with $10 \%$ (blue) and $60 \%$ (red) of the maximum value.

given in Ref. ${ }^{12}$. In step two $\left(\Delta_{\text {so }}=0, \Delta_{\text {cf }} \neq 0\right)$ the parameter $E_{p_{z}, p_{z}}^{A}$ is included in the TB-model to reproduce the splitting of the $A-C$ bands. Of course all the other matrix elements are also re-adjusted to obtain the correct band structure. Starting from these TB parameters, in the final step $\left(\Delta_{\text {so }} \neq 0, \Delta_{\text {cf }} \neq 0\right)$ the additional parameter $\lambda$ is taken into account to reproduce the splitting of the bands $A-B$. Again, all parameters are re-adjusted to reproduce the correct energetic positions of the different bands. The resulting parameters are given in Tab. [, Figure 3 shows the QD geometry and first three bound one-particle states for electrons and holes, respectively, including the influence of the built-in field.

According to the nodal structure, the depicted electron 
ground state $\psi_{1}^{e}$ can be classified as $s$-like. The first two excited states $\psi_{2}^{e}$ and $\psi_{3}^{e}$ can be classified as $p_{+}$and $p_{-}$ states, respectively. Such a classification is not possible for the hole states, since these states undergo strong band mixing effects. Considering only a single valence band for the description of the bound hole states in an InN QD is not valid. The observation of strong band mixing effects is in agreement with other multi-band approaches. $5,16,31$ From Fig. 3 we can deduce that CF splitting and SO coupling do not alter the single-particle level structure. In other words, for the ordering of the first three bound electron and hole states in a lens-shaped InN QD, the contributions from SO and CF splitting are negligible.

After this discussion of the single-particle states we focus on the single-particle energies of the large QD. In Tab. III the energies of the first three bound electron and hole states under the influence of the built-in field are displayed. From this table we conclude that the electron states are only slightly shifted to lower energies by CF splitting and SO coupling. Without SO coupling $\left(\Delta_{\text {so }}=0\right)$, and taking only $\mathrm{CF}$ splitting into account $\left(\Delta_{\mathrm{cf}} \neq 0\right)$, the hole states are shifted to higher energies. With SO coupling, the hole energy spectrum is shifted to lower energies, compared to the case neglecting both contributions $\left(\Delta_{\mathrm{cf}}=0, \Delta_{\text {so }}=0\right)$. Additionally it turns out that the degeneracy of the hole states $\psi_{2}^{h}$ and $\psi_{3}^{h}$ ( $p$-shell) is lifted when SO coupling is considered. Of course, each state is still twofold degenerate due to time reversal symmetry ${ }^{33}$. Because of the small SO energies of the bulk materials, the splitting is rather small $\left(\Delta_{\psi_{1}^{h}, \psi_{2}^{h}}=1.2 \mathrm{meV}\right)$. The same is true for the electron $p$-states. Here the influence of the SO coupling is even weaker $\left(\Delta_{\psi_{1}^{h}, \psi_{2}^{h}}=0.1 \mathrm{meV}\right)$.

As discussed recently, 13 , for the system under investigation, a TB model which neglects the weak crystal-field splitting and spin-orbit coupling, must result in degenerate $p$-shells for electrons and holes. The origin of these degeneracies is the $C_{3 v}$ symmetry of the combined system of QD geometry and underlying wurtzite lattice.

From the splitting of the hole states $\psi_{2}^{h}$ and $\psi_{3}^{h}$, one can deduce that the spin-orbit interaction alters the symmetry of the system. This can be understood by an analysis of the corresponding character tables for $C_{3 v}$ single and double groups.

Without spin-orbit coupling, the symmetry of the system is determined by the single group $C_{3 v}$. Looking at the character table, shown in Tab. III we conclude that this group allows for double degenerate levels, since the group contains a two-dimensional representation $\Gamma_{3}$. One

TABLE II: Character table for the single group $C_{3 v}$ (Ref. $\left.{ }^{32}\right)$.

\begin{tabular}{|c|ccc|}
\hline \hline & $\{E\}$ & $\left\{2 C_{3}\right\}$ & $\left\{3 \sigma_{v}\right\}$ \\
\hline$\Gamma_{1}$ & 1 & 1 & 1 \\
$\Gamma_{2}$ & 1 & 1 & -1 \\
$\Gamma_{3}$ & 2 & -1 & 0 \\
\hline \hline
\end{tabular}

TABLE III: Single-particle energies for the large InN QD in the presence and absence of crystal field splitting and spinorbit coupling. Each of the given states is two-fold degenerate due to time reversal symmetry.

\begin{tabular}{|c|c|c|c|}
\hline \hline & $\Delta_{\text {so }}=0, \Delta_{\text {cf }}=0$ & $\Delta_{\text {so }}=0, \Delta_{\text {cf }} \neq 0$ & $\Delta_{\text {so }} \neq 0, \Delta_{\text {cf }} \neq 0$ \\
\hline$E_{1}^{e}[\mathrm{eV}]$ & 1.4770 & 1.4585 & 1.4557 \\
$E_{2}^{e}[\mathrm{eV}]$ & 1.6660 & 1.6464 & 1.6417 \\
$E_{3}^{e}[\mathrm{eV}]$ & 1.6660 & 1.6464 & 1.6418 \\
\hline$E_{1}^{h}[\mathrm{eV}]$ & 0.9021 & 0.9041 & 0.8993 \\
$E_{2}^{h}[\mathrm{eV}]$ & 0.9021 & 0.9041 & 0.8981 \\
$E_{3}^{h}[\mathrm{eV}]$ & 0.8964 & 0.8989 & 0.8924 \\
\hline \hline
\end{tabular}

example of such degenerate states are the $p$-shell states for electrons $\left(\psi_{2}^{e} ; \psi_{3}^{e}\right)$ and holes $\left(\psi_{1}^{h} ; \psi_{2}^{h}\right)$, shown in Fig. 3 . Of course, in the absence of the SO coupling, in addition, each state is two-fold spin degenerate.

Including SO coupling, one has to deal with the double group $\bar{C}_{3 v}$. The character table of the double group is given in Tab. IV] This group allows only two dimensional representations, even if the time reversal symmetry is included ${ }^{33}$. More specifically, the degeneracy of the irreducible representations $\Gamma_{3}$ and $\Gamma_{4}$ is not doubled by the time reversal symmetry ${ }^{33}$ In other words, no fourfold degenerate state in the energy spectrum can exist. Consequently, the electron $p$ states $\left(\psi_{2}^{e}\right.$ and $\left.\psi_{3}^{e}\right)$ are also no longer degenerate, but in this particular case the splitting is only of the order of some $\mu \mathrm{eV}$. We note that this is in contrast to the findings in Ref. ${ }^{16}$, where (at least numerically) exact degeneracy of the electronic $p$-states has been reported.

A central result of the previous work ${ }^{12,13}$ was that the strong internal electrostatic field can reverse the energetic ordering of the first three bound hole states. We find that, for the intermediate and the largest InN QD, in the presence of the built-in field, the ground state is formed by the twofold degenerate $p$ states $\psi_{1}^{h}$ and $\psi_{2}^{h}$, shown in Fig. 3. This behavior is interchanged with decreasing QD size, where, for the smallest QD, the $s$ state $\psi_{3}^{h}$ becomes the hole ground state. To concentrate on this reordering of the hole $s$ and $p$ shell, the energy splitting $\Delta E_{s, p}^{h}=E_{s}^{h}-E_{p}^{h}$ is displayed in Fig. 4. In order to analyze the impact of $\mathrm{CF}$ splitting and SO coupling on the ordering of the hole level structure, we calculate the

TABLE IV: Character table for the double group $\bar{C}_{3 v}$ (Ref. $\left.{ }^{34}\right)$.

\begin{tabular}{|c|cccccc|}
\hline \hline & $\{E\}$ & $\{\bar{E}\}$ & $\left\{2 C_{3}\right\}$ & $\left\{2 \bar{C}_{3}\right\}$ & $\left\{3 \sigma_{v}\right\}$ & $\left\{3 \bar{\sigma}_{v}\right\}$ \\
\hline$\Gamma_{1}$ & 1 & 1 & 1 & 1 & 1 & 1 \\
$\Gamma_{2}$ & 1 & 1 & 1 & 1 & -1 & -1 \\
$\Gamma_{3}$ & 2 & 2 & -1 & -1 & 0 & 0 \\
$\Gamma_{4}$ & 2 & -2 & 1 & -1 & 0 & 0 \\
$\Gamma_{5}$ & 1 & -1 & -1 & 1 & $i$ & $-i$ \\
$\Gamma_{6}$ & 1 & -1 & -1 & 1 & $-i$ & $i$ \\
\hline \hline
\end{tabular}




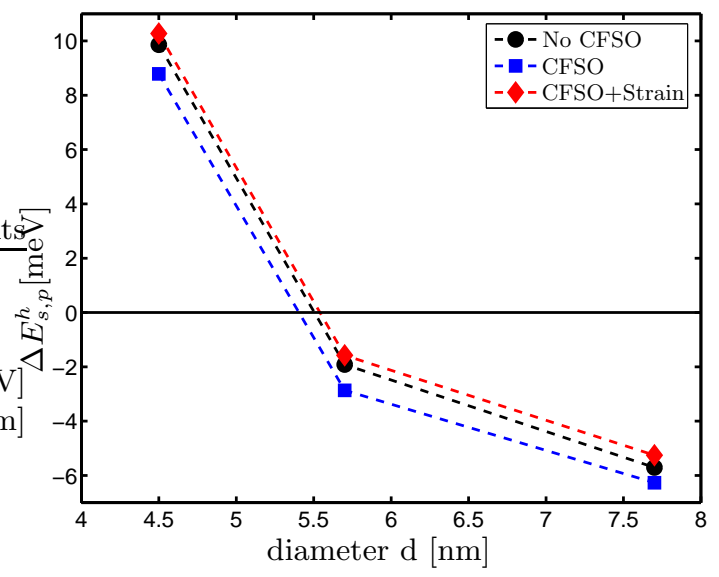

FIG. 4: (Color online) The energy splitting $\Delta E_{s, p}^{h}=E_{s}^{h}-$ $E_{p}^{h}$ between the $s$ and the $p$ shell for the holes is shown in the absence (No CFSO) and presence (CFSO) of crystal field $\left(\Delta_{\mathrm{cf}}\right)$ and spin-orbit $\left(\Delta_{\text {so }}\right)$ splitting. Results are also shown, where in addition to crystal field and spin-orbit splitting a strain field is included (CFSO+Strain). The influence of the strain field on the electronic properties is discussed in detail in Sec. IIIB. In all three cases, $\Delta E_{s, p}^{h}$ changes sign with increasing QD diameter $d$, as a level reordering occurs (the dashed lines are included as a guide to the eye). The built-in field is included in all the calculations.

splitting $\Delta E_{s, p}^{h}$ when both contributions are introduced in the TB approach. Since the hole $p$ shell is no longer degenerate, we average over the single-particle energies of the states $\psi_{1}^{h}$ and $\psi_{2}^{h}$. The energy splitting $\Delta E_{s, p}^{h}$ with SO coupling and CF splitting is also shown in Fig. 4. From the comparison with $\Delta E_{s, p}^{h}$ in the absence of these contributions, we find that the SO coupling and the CF splitting have only a negligible effect on the energy splitting $\Delta E_{s, p}^{h}$. Furthermore, the ordering of the first three bound hole states is unaffected by $\mathrm{SO}$ coupling and $\mathrm{CF}$ splitting.

In summary, the CF splitting alone cannot alter the symmetry of the system and leads only to a small energy shift of the first three bound electron and hole states. The SO interaction, and only this contribution, can modify the symmetry, and lifts certain degeneracies. However, the splitting of the electron and hole $p$ shell due to the SO coupling, is very small compared to the level spacing of the different shells. Moreover, in the presence of SO coupling and CF splitting, one obtains the same level ordering of the energetically lowest hole states ( $s$ - and $p$-shell) as in the case were these contributions are not taken into account. This analysis indicates that it is well justified to neglect these small corrections of the CF splitting and the SO coupling in the system under consideration.

\section{B. Influence of strain}

So far we have neglected the influence of the lattice mismatch between InN and GaN. The lattice mismatch
TABLE V: Material parameters for GaN and InN. If not indicated otherwise, all parameters are taken from Ref. ${ }^{35}$.

\begin{tabular}{|c|c|c|}
\hline Parameter & $\mathrm{GaN}$ & $\operatorname{InN}$ \\
\hline $\mathrm{a}(\AA)$ & 3.189 & 3.545 \\
\hline c $(\AA)$ & 5.185 & 5.703 \\
\hline$\Delta_{\text {so }}(\mathrm{eV})$ & $0.010\left(\operatorname{Ref}^{3}{ }^{3}\right)$ & $0.005\left(\right.$ Ref $\left.^{3}\right)$ \\
\hline$\Delta_{\mathrm{cf}}(\mathrm{eV})$ & $0.017\left(\operatorname{Ref}^{3}{ }^{3}\right)$ & $0.040\left(\right.$ Ref $\left.^{3}\right)$ \\
\hline$C_{13}(\mathrm{GPa})$ & 11.4 & 9.4 \\
\hline$C_{33}(\mathrm{GPa})$ & 38.1 & 20.0 \\
\hline$\left(a_{c}-D_{1}\right)(\mathrm{eV})$ & $-9.6\left(\operatorname{Ref}^{36}\right)$ & $-9.6\left(\operatorname{Ref}^{36}\right)$ \\
\hline$\left(a_{c}-D_{2}\right)(\mathrm{eV})$ & $-8.2\left(\operatorname{Ref}^{36}\right)$ & $-8.2\left(\operatorname{Ref}^{36}\right)$ \\
\hline$D_{3}(\mathrm{eV})$ & $1.9\left(\right.$ Ref $\left.^{36}\right)$ & $1.9\left(\right.$ Ref $\left.^{36}\right)$ \\
\hline$D_{4}(\mathrm{eV})$ & $-1.0\left(\operatorname{Ref}^{36}\right)$ & $-1.0\left(\right.$ Ref $\left.^{36}\right)$ \\
\hline$a_{c}(\mathrm{eV})$ & $-4.9\left(\right.$ Ref $\left.^{3}\right)$ & $-3.5\left(\right.$ Ref $\left.^{3}\right)$ \\
\hline
\end{tabular}

leads to the appearance of a strain field in the nanostructure. This field modifies the energies of the bound electron and hole states.

For the electron states, caused by the underlying wurtzite lattice and the assumed QD geometry, the strain field produces only an energy shift of the bound single particle states ${ }^{7}$. In contrast to QDs with a zinc blende structure $\frac{15}{}$, no degeneracies are lifted by the strain field. The situation is more complicated for the hole states. The strain field modifies the local valence band edges, and can therefore lead to a splitting of the different energy bands. As discussed in Ref $\frac{16}{16}$, due to the biaxial strain in the basal plane, the first two valence bands ( $A$ and $B$ ) are shifted to higher energies, compared to the unstrained material, whereas the third valence band $(C$ band) is shifted to lower energies. These energy shifts may also increase the influence of spin-orbit coupling and crystal field splitting on the bound single particle states.

To investigate the influence of these shifts and the effect of the possible valence band splittings, we proceed in the following way:

Since the TB parameters are fitted to the bulk band structure, we re-calculate the bulk band structure of InN in the presence of a strain field. To obtain the strain dependent valence band edge we apply ${ }^{37}$

$$
\begin{aligned}
E_{1}= & \Delta_{\mathrm{cf}}+\frac{1}{3} \Delta_{\mathrm{so}}+\theta_{\epsilon}+\lambda_{\epsilon}, \\
E_{2,3}= & \frac{\Delta_{\mathrm{cf}}}{2}-\frac{\Delta_{\mathrm{so}}}{3}+\frac{\theta_{\epsilon}}{2}+\lambda_{\epsilon} \\
& \mp \sqrt{\left(\frac{\Delta_{\mathrm{cf}}-\frac{\Delta_{\mathrm{so}}}{3}+\theta_{\epsilon}}{2}\right)^{2}+\frac{2}{9}\left(\Delta_{\mathrm{so}}\right)^{2}},
\end{aligned}
$$

where $\theta_{\epsilon}$ and $\lambda_{\epsilon}$ are given by

$$
\begin{aligned}
& \theta_{\epsilon}=D_{3} \epsilon_{z z}+D_{4}\left(\epsilon_{x x}+\epsilon_{y y}\right), \\
& \lambda_{\epsilon}=D_{1} \epsilon_{z z}+D_{2}\left(\epsilon_{x x}+\epsilon_{y y}\right) .
\end{aligned}
$$

Here, $D_{1}, D_{2}, D_{3}$ and $D_{4}$ are the valence band deformation potentials and $\epsilon_{i i}$ denotes the diagonal components 


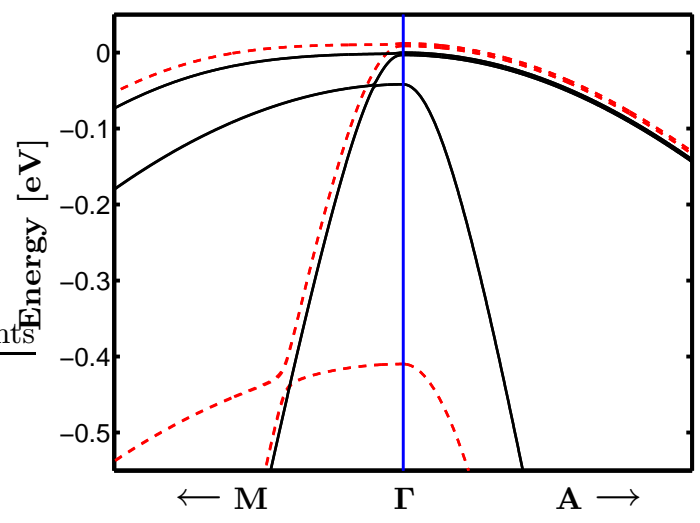

FIG. 5: (Color online) Calculated TB valence band structure in the vicinity of the $\Gamma$ point. Unstrained (black solid lines) and under compressive biaxial strain in the basal plane with elastic relaxation along the [0001] direction (red dashed lines). The splitting between the $A$ and $B$ valence bands at the $\Gamma$ point is $\approx 3.1 \mathrm{meV}(\approx 3.1 \mathrm{meV})$ in unstrained (strained) InN.

of the strain tensor $\underline{\underline{\epsilon}}$. For the components $\epsilon_{i i}$ we assume:

$$
\epsilon_{z z}=\frac{a_{0}-a}{a}, \epsilon_{x x}=\epsilon_{y y}=-\frac{2 C_{13}}{C_{33}} \epsilon_{x x},
$$

where $a_{0}$ and $a$ are the lattice constants of the substrate and the QD material, respectively, and $C_{13}$ and $C_{33}$ are the stiffness constants. Strictly speaking, these equations apply only to a quantum well, since they neglect shear strain components and the fact that the strain field in a QD is position dependent. However, due to the symmetry of the QD structure and the underlying wurtzite lattice, the strain field can not lift degeneracies. This is also confirmed by results of Winkelnkemper et. $a l^{16}$ for an $\mathrm{In}_{x} \mathrm{Ga}_{1-x} \mathrm{~N}$ QD with a comparable symmetry. Furthermore, the hole states, for which the modification of the valence band edge is of major importance, are strongly localized in the region of the QD. Therefore, the variation of the strain field outside the QD is of minor importance. In the region of the InN QD, we use the lattice constant $a$ of GaN, to take into account, that the QD is pseudomorphically grown on the GaN substrate.

According to Ref ${ }^{37}$, the band gap shift is given by

$$
\Delta E^{\text {gap }}=E_{0}^{\text {gap }}+P_{c \epsilon}-\left(\theta_{\epsilon}+\lambda_{\epsilon}\right)
$$

with

$$
\begin{aligned}
& P_{c \epsilon}=a_{c} \epsilon_{z z}+a_{c}\left(\epsilon_{x x}+\epsilon_{y y}\right), \\
& \lambda_{\epsilon}=D_{1} \epsilon_{z z}+D_{2}\left(\epsilon_{x x}+\epsilon_{y y}\right),
\end{aligned}
$$

where $a_{c}$ denotes the conduction band deformation potential. The different parameters are listed in Tab. V The TB parameters are readjusted to reproduce the bulk band structure in the vicinity of the $\Gamma$ point. The resulting valence band structure in the vicinity of the $\Gamma$ point is depicted in Fig. 5. The band structure exactly reflects the behavior which is expected for the local band structure in the region of the QD: $: \underline{16}$ the first two valence bands
TABLE VI: Single-particle energies for the large InN QD in

\begin{tabular}{|c|c|c|}
\hline & \multicolumn{2}{|c|}{$\Delta_{\text {so }} \neq 0, \Delta_{\text {cf }} \neq 0$} \\
\hline & Without Strain & With Strain \\
\hline$E_{1}^{e}[\mathrm{eV}]$ & 1.4557 & 1.7740 \\
\hline$E_{2}^{e}[\mathrm{eV}]$ & 1.6417 & 1.9625 \\
\hline$E_{3}^{e}[\mathrm{eV}]$ & 1.6418 & 1.9626 \\
\hline$E_{1}^{h}[\mathrm{eV}]$ & 0.8993 & 0.9126 \\
\hline$E_{2}^{h}[\mathrm{eV}]$ & 0.8981 & 0.9118 \\
\hline$E_{3}^{h}[\mathrm{eV}]$ & 0.8924 & 0.9069 \\
\hline
\end{tabular}
the presence and absence of strain effects. Each of the given states is two-fold degenerate due to time reversal symmetry. The internal electrostatic field is included in the calculation.

$(A$ and $B)$ are shifted to higher energies while the third band $(C)$ is shifted to lower energies. The calculated TB parameters are now used to investigate the influence of strain effects on the electronic states.

Starting from these new TB parameters one can recalculate the single-particle states and energies of the three different QDs discussed in the preceding section, taking into account both $\mathrm{CF}$ and SO splitting. First we focus on the single-particle energies of the large QD $(d=7.7 \mathrm{~nm}, h=3.0 \mathrm{~nm})$. In Tab. VI, the energies of the first three bound electron and hole states, including the built-in field are displayed. Each of the given states is two-fold degenerate due to time reversal symmetry. The results in the absence and in the presence of the strain effects are compared. First of all, the strain merely produces an energy shift of the single particle states. The strain field shifts both electron states and hole states to higher energies. This behavior reflects the local band edge shifts of conduction and valence bands. Following the discussion of the previous section, no four-fold degenerate states can exist taking SO coupling into account. As already discussed, the first two excited states $E_{2}^{e}$ and $E_{3}^{e}$ are nearly degenerate in the absence of the strain field. Also in the presence of the strain field, these states are split by the SO coupling by $0.1 \mathrm{meV}$ only. This analysis shows that the splitting of the electron $p$-states is not altered by the strain field and remains very small compared to the energy separation of electron $s-\left(\psi_{1}^{e}\right)$ and $p$-shell $\left(\psi_{2}^{e}\right.$ and $\left.\psi_{3}^{e}\right)$. The splitting of the hole states $\psi_{1}^{h}$ and $\psi_{2}^{h}$ is nearly unaffected by the strain field.

Additionally, the level ordering is not modified by the strain field. In the presence of the strain field, the intrinsic electrostatic field still reverses the energetic ordering of the first three bound hole states. The energy splitting $\Delta_{s, p}^{h}$ between the hole $s$ and $p$ shell including the strain field is displayed in Fig. 4. We compare these results with the other results shown in Fig. (4) where the strain field is absent. In conclusion, the strain field is of minor importance for the energy splittings, and, most importantly, the ordering of the first three bound hole states is unaffected. 


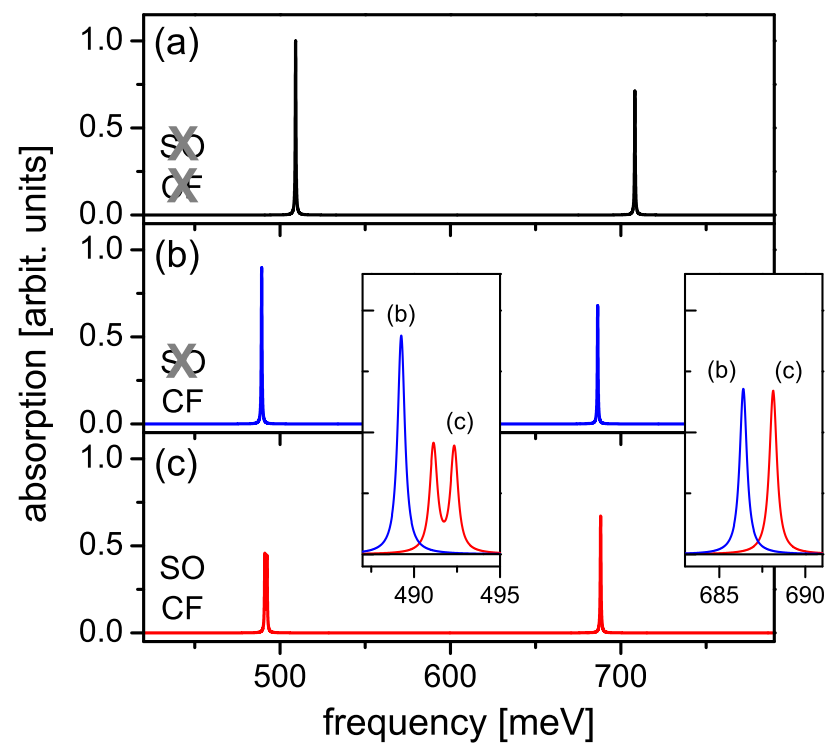

FIG. 6: (Color online) Excitonic absorption spectra for the largest InN QD in the absence of crystal field and spin-orbit splitting (a), in the presence of crystal field splitting and absence of spin-orbit coupling (b) and in the presence of crystalfield and spin-orbit splitting (c). The insets show the same data for frequencies close to the absorption peaks in (b) and (c).

\section{Excitonic absorption spectra}

In Sec. II C we have discussed the calculation of dipole and Coulomb matrix elements. The evaluation of excitonic absorption spectra in this section can be performed starting from the many-particle Hamiltonian, Eq. (2), in second quantization as given in Sec. IIC. For the localized states configuration-interaction calculations are performed. For the sake of simplicity, only the first three bound electron and hole states are included. This can be justified by their energy separation to higher shells in the structure. The excitonic absorption spectra are calculated using Fermi's golden rule ${ }^{30}$

Figure 6 (a) shows the excitonic absorption spectrum for the largest InN QD in the absence of CF and SO splitting, while Fig. 6 (b) displays the spectrum in presence of the CF splitting but in the absence of SO coupling. In Fig. 6 (c), the absorption spectrum in the presence of both CF and SO splitting is depicted. The different absorption lines in each spectrum correspond to the excitation of an exciton in the QD. In case (a) and (b) the peak on the low energy side corresponds to transitions where the electron is mainly in the ground state $\psi_{1}^{e}$, and the hole is mainly in the states $\psi_{1}^{h}$ and $\psi_{2}^{h}$. Since the hole states $\psi_{1}^{h}$ and $\psi_{2}^{h}$ are degenerate one obtains only a single peak on the low energy side. Due to the SO coupling, the states $\psi_{1}^{h}$ and $\psi_{2}^{h}$ are split by about $1.2 \mathrm{meV}$. This splitting in the single particle states results in two lines on the low energy side in Fig. 6 (c) (see also the inset). Since the splitting in the single-particle states $\psi_{1}^{h}$ and $\psi_{2}^{h}$ is very small, the splitting of the two peaks on the low energy side is also very small. This emphasizes again, that in the system under consideration the SO coupling and the CF splitting introduce only negligible corrections to the excitonic spectrum. Due to the symmetry of the QD and the underlying wurtzite structure, there is no polarization anisotropy. This is in contrast to lens-shaped InAs QDs with a zinc blende structure, as discussed in Ref $\stackrel{38}{ }$, where the $C_{2 v}$ symmetry leads to polarization anisotropy.

The peak on the high energy side mainly corresponds to the excitation of the hole in the state $\psi_{3}^{h}$ and the states $\psi_{2}^{e}$ and $\psi_{3}^{e}$. Since the electron states $\psi_{2}^{e}$ and $\psi_{3}^{e}$ are exactly degenerate in cases (a) and (b) and nearly degenerate in case (c), only a single line is visible on the high energy side (cf. inset of Fig. 6) ).

Including strain effects, we obtain nearly the same splitting of the states $\psi_{1}^{h}$ and $\psi_{2}^{h}$. Furthermore, the electron states $\psi_{2}^{e}$ and $\psi_{3}^{e}$ are still nearly degenerate. Only the single-particle energy gap is enlarged by the strain field and therefore the whole excitonic absorption spectrum is shifted to higher energies. The excitonic absorption spectrum (not shown) resembles the spectrum in Fig. 6] (c).

\section{CONCLUSION}

In this work we have presented an atomistic TB calculation of the electronic and optical properties of lensshaped InN QDs. We focused our attention on the influence of the crystal-field splitting and the spin-orbit coupling on the electronic structure as well as on the optical properties. As it turns out, only the spin-orbit coupling lifts certain degeneracies in the single particle spectrum. This result is confirmed by the inspection of the character table of the double group $\bar{C}_{3 v}$, which reveals that no four fold degenerate state can exist. However, from our calculations we obtain only small splittings in the singleparticle spectrum as well as in the excitonic absorption spectrum. Our results indicate that it is a reasonable assumption to neglect spin-orbit coupling and crystal field splitting in case of lens-shaped InN QDs with a wurtzite structure.

We do not rule out that the importance of spinorbit coupling and crystal-field splitting may depend on the specific system under investigation even within the group-III nitride material system where both effects are intrinsically weak.

\section{Acknowledgments}

We thank Paul Gartner, Norman Baer, and Frank Jahnke for various helpful discussions. This work has been supported by the Deutsche Forschungsgemeinschaft (research group "Physics of nitride-based, nanostructured, light-emitting devices", project $\mathrm{Cz}$ 
31/14-1,2). S. Schumacher was further supported by the Deutsche Forschungsgemeinschaft through project No. SCHU 1980/3-1. We also acknowledge a grant for CPU time from the NIC at the Forschungszentrum Jülich.
1 O. Ambacher, J. Phys. D: Appl. Phys. 31, 2653 (1998).

2 A. G. Bhuiyan, A. Hashimoto, and A. Yamamoto, J. Appl. Phys. 94, 2779 (2003).

3 I. Vurgaftman and J. R. Meyer, J. Appl. Phys. 94, 3675 (2003).

4 P. Michler, A. Kiraz, C. Becher, W. V. Schoenfeld, P. M. Petroff, L. Zhang, E. Hu, and A. Imamoglu, Science 290, 2282 (2000).

5 A. D. Andreev and E. P. O'Reilly, Phys. Rev. B 62, 15851 (2000).

6 A. D. Andreev and E. P. O'Reilly, Appl. Phys. Lett. 79, 521 (2001).

7 V. A. Fonoberov and A. A. Baladin, J. Appl. Phys. 94, 7178 (2003).

8 T. Saito and Y. Arakawa, phys. stat. sol. (c) 0, 1169 (2003).

9 T. Saito and Y. Arakawa, Journal of Crystal growth 237, 1172 (2002).

10 T. Saito and Y. Arakawa, Physica E (Amsterdam) 15, 169 (2002).

11 N. Baer, S. Schulz, S. Schumacher, P. Gartner, G. Czycholl, and F. Jahnke, Appl. Phys. Lett. 87, 231114 (2005).

12 S. Schulz, S. Schumacher, and G. Czycholl, Phys. Rev. B 73, 245327 (2006).

13 N. Baer, S. Schulz, P. Gartner, S. Schumacher, G. Czycholl, and F. Jahnke, Phys. Rev. B 76, 075310 (2007).

14 S. Schulz, S. Schumacher, and G. Czycholl, phys. stat. sol. b 244, 2399 (2007).

15 G. Bester and A. Zunger, Phys. Rev. B 71, 045318 (2005).

16 M. Winkelnkemper, A. Schliwa, and D. Bimberg, Phys. Rev. B 74, 155322 (2006).

17 A. Kobayashi, O. F. Sankey, S. M. Volz, and J. D. Dow, Phys. Rev. B 28, 935 (1983).

18 D. J. Chadi, Phys. Rev. B 16, 790 (1977).

19 A. Yoshikawa and K. Xu, Thin Solid Films 412, 38 (2002).

20 M. Murayama and T. Nakayama, Phys. Rev. B 49, 4710 (1994).

21 D. Fritsch, H. Schmidt, and M. Grundmann, Phys. Rev. B
69, 165204 (2004).

22 G. L. Zhao, D. Bagayoko, and T. D. Williams, Phys. Rev. B 60, 1563 (1999).

23 M. Grundmann, The Physics of Semiconductors: An Introduction Including Devices and Nanophysics (Springer, Berlin, 2006).

24 J.-M. Jancu, F. Bassani, F. D. Salla, and R. Scholz, Appl. Phys. Lett. 81, 4838 (2002).

25 P. Carrier and S.-H. Wei, J. Appl. Phys. 97, 033707 (2005).

26 S. B. S. K. Pugh, D. J. Dugdale and R. A. Abram, Semicond. Sci. Technol. 14, 23 (1999).

27 F. Bernardini, V. Fiorentini, and D. Vanderbilt, Phys. Rev. B 56, R10024 (1997).

28 S. De Rinaldis, I. D'Amico, and F. Rossi, Phys. Rev. B 69, 235316 (2004).

29 J. C. Slater and G. F. Koster, Phys. Rev. 94, 1498 (1954).

${ }^{30}$ N. Baer, P. Gartner, and F. Jahnke, Eur. Phys. J. B 42, 231 (2004).

31 V. A. Fonoberov and A. A. Balandin, J. Vac. Sci. Technol. B 22, 2190 (2004).

32 J. F. Cornwell, Group Theory and electronic energy bands in solids (North-Holland Pubishing Company, Amsterdam, 1969).

33 F. Bassani and G. P. Parravicini, Electronic states and optical transitions in solids (Pergamon Press, New York, 1975).

34 E. Kartheuser and S. Rodriguez, Phys. Rev. B 8, 1556 (1973).

35 J.-J. Shi and Z.-Z. Gan, J. Appl. Phys. 94, 407 (2003).

${ }^{36}$ H. Y. Peng, M. D. McCluskey, Y. M. Gupta, M. Kneissl, and N. M. Johnson, Phys. Rev. B 71, 115207 (2005).

37 S. L. Chuang and C. S. Chang, Phys. Rev. B 54, 2491 (1996).

38 A. J. Williamson, L. W. Wang, and A. Zunger, Phys. Rev. B 62, 12963 (2000). 